\title{
Effect of $\alpha-\mathrm{Hgl}_{2}$ epitaxial growth on the defect structure of CdTe:Ge substrates
}

\author{
G. Panin a) and J. Piqueras \\ Departamento de Fisica de Materiales, Facultad de Fisicas, Universidad Complutense, 28040 Madrid, \\ Spain \\ N. Sochinskiib),c) and E. Dieguez \\ Departamento de Fisica de Materiales, Universidad Autonoma, 28049 Madrid, Spain
}

(Received 19 February 1996; accepted for publication 10 December 1996)

\begin{abstract}
The $\alpha-\mathrm{HgI}_{2} / \mathrm{CdTe}: \mathrm{Ge}$ heterostructures have been studied by cathodoluminescence (CL) in the scanning electron microscope. The $\alpha-\mathrm{HgI}_{2}$ expitaxial growth was shown to cause an enhancement of the CL intensity in a layer of the substrate extending up to about $500 \mu \mathrm{m}$ from the $\alpha-\mathrm{HgI}_{2} / \mathrm{CdTe}: \mathrm{Ge}$ interface. CL spectra of the layer reveal the appearance of a band related to tellurium vacancies as well as the decrease of the emission attributed to defect complexes involving $\mathrm{Ge}$. The data obtained indicate that Ge-impurity gettering and $V_{\mathrm{Te}}$ generation at the interface take place during $\alpha-\mathrm{HgI}_{2}$ epitaxial growth. (C) 1997 American Institute of Physics.
\end{abstract}

[S0003-6951(97)02807-6]

The compound semiconductors $\alpha-\mathrm{HgI}_{2}$ and CdTe are raising considerable interest for their potential applications ${ }^{1-5}$ in room temperature $\mathrm{x}$-ray and $\gamma$-ray detectors due to the wideband gaps, large average atomic masses, and high resistivity (after suitable doping with the groups IV or VII elements in the case of $\mathrm{CdTe}^{6}$ ). Taking into account the difference of the band gap values of both materials (2.3 $\mathrm{eV}$ for $\alpha-\mathrm{HgI}_{2}$ and $1.5 \mathrm{eV}$ for CdTe at $300 \mathrm{~K}$ ), the growth of $\alpha-\mathrm{HgI}_{2} / \mathrm{CdTe}$ heterostructures would be of interest for the fabrication of detectors operating in an extended wavelength range.

Recently, the cathodoluminescence (CL) technique has been shown to provide important data on the behavior of the structural defects controlling optical and electrical properties of both CdTe and $\alpha-\mathrm{HgI}_{2}$ bulk crystals. ${ }^{7-9}$ However, very limited attention has been given to the study of luminescence properties of high-resistivity doped $\mathrm{CdTe}$ and epitaxial structures made from it. In the particular case of CdTe:Ge, which is the substrate used in this work, previous CL studies ${ }^{8}$ show an enhancement of 810 and $910 \mathrm{meV}$ emissions as a result of Ge doping. Both bands show an increase in intensity after an annealing of the Ge-doped sample which is attributed to the formation of a complex center involving $\mathrm{Ge}$ on $\mathrm{Cd}$ sites. No near band-edge emission is observed in Ge-doped samples. In the present letter we report on a study by CL of the defect structure of CdTe:Ge substrate affected by $\alpha-\mathrm{HgI}_{2}$ epitaxial growth. This spatial resolved technique enables us to study the nature and extension of the changes induced in the substrate by the growth.

Ge-doped $\left(10^{19}\right.$ atoms $\left.\mathrm{cm}^{-3}\right)$ CdTe single crystals grown by the vertical Bridgman method with a room temperature resistivity of $10^{9} \Omega \mathrm{cm}$ were used. Some $\alpha-\mathrm{HgI}_{2}$ layers 20-30 $\mu \mathrm{m}$ thick were grown on the (110) CdTe surface by isothermal vapor phase epitaxy at $250{ }^{\circ} \mathrm{C}$ for $24 \mathrm{~h}$ as

${ }^{a)}$ Permanent address: Institute of Microelectronics Technology and High Purity Materials, Russian Academy of Sciences, Chernogolovka, Moscow district 142432, Russia.

${ }^{b}$ Institute of Semiconductor Physics, Pr. Nauki 45, 252650, Kiev, Ukraine.

${ }^{c}$ New Semiconductors, Inc., 254210, Kiev, Ukraine. described elsewhere. ${ }^{11} \mathrm{CL}$ measurements were carried out on cross sections of the cleaved $\alpha-\mathrm{HgI}_{2} / \mathrm{CdTe}$ structures in a Hitachi S-2500 scanning electron microscope at $80 \mathrm{~K}$ with $25 \mathrm{keV}$ accelerating voltage, by using a liquid nitrogen cooled germanium detector. Details of the CL experimental setup have been previously described. ${ }^{12}$

Figure 1 shows representative secondary electron (a) and CL (b) images of a cross section of the $\alpha-\mathrm{HgI}_{2} / \mathrm{CdTe}: \mathrm{Ge}$ heterostructure near the interface as observed on several

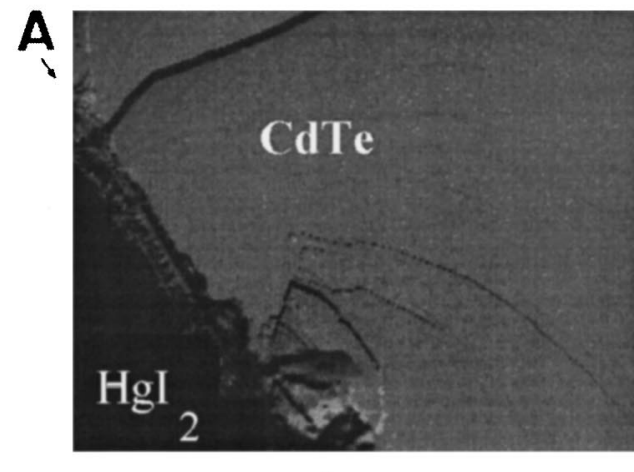

'A

(a)

$100 \mu \mathrm{m}$

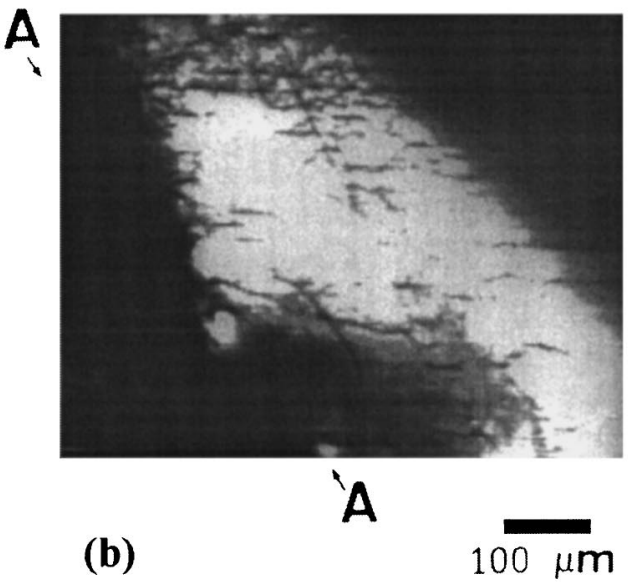

FIG. 1. (a) SE image of cleaved $\alpha-\mathrm{HgI}_{2} / \mathrm{CdTe}$ Ge structure, (b) CL image of the same region. Line A-A marks the $\alpha-\mathrm{HgI}_{2} / \mathrm{CdTe}$ :Ge interface. 


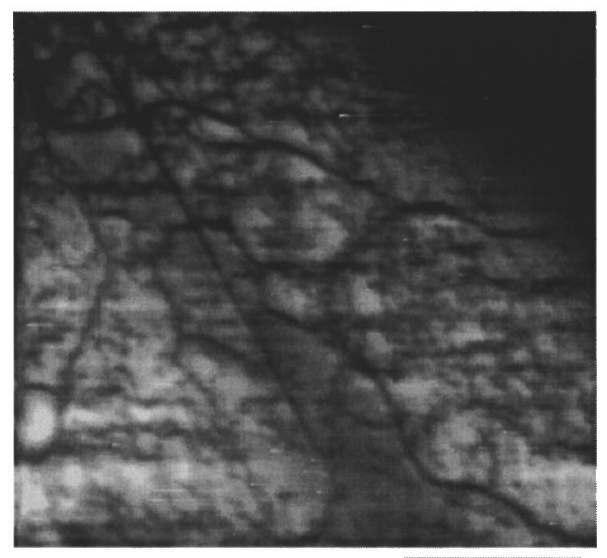

(a)

$100 \mu \mathrm{m}$

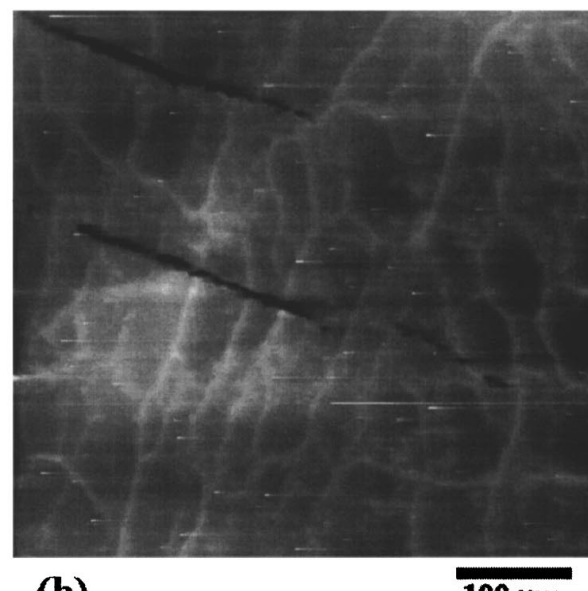

(b)

$100 \mu \mathrm{m}$

FIG. 2. CL images of a modified layer (a) and the substrate bulk region far from this layer (b).
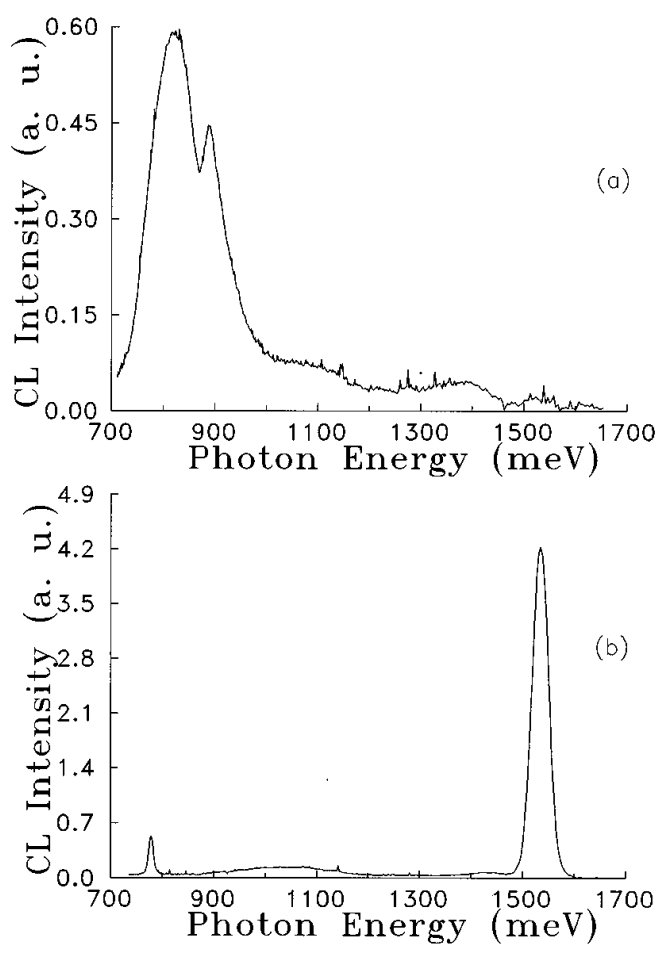

FIG. 3. CL spectra recorded with a focused electron beam in scan mode from a large $(300 \mu \mathrm{m} \times 300 \mu \mathrm{m})$ area in the substrate bulk region far from the modified layer (a) and inside the modified layer (b).
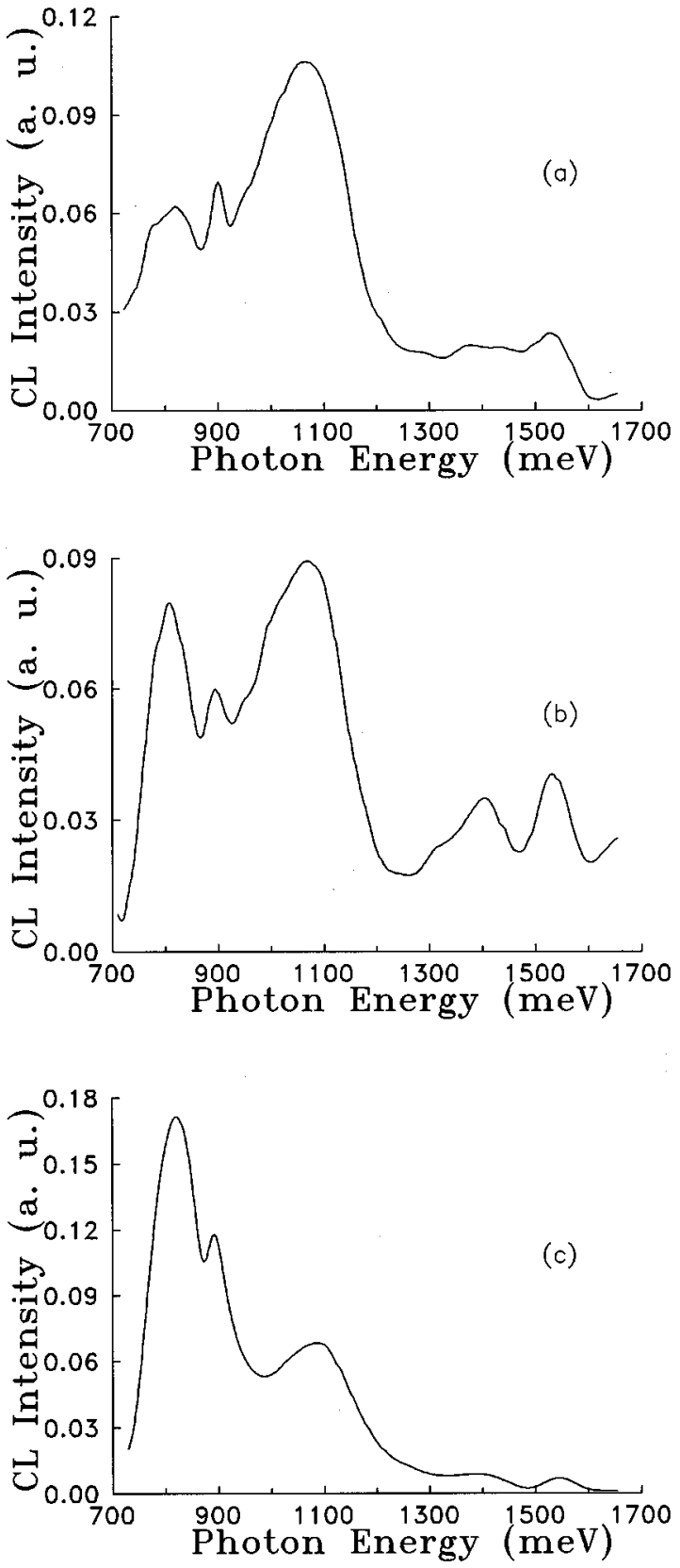

FIG. 4. CL spectra of the substrate recorded with a defocused electron beam in scan mode from an area of about $50 \mu \mathrm{m} \times 50 \mu \mathrm{m}$ positioned on sub grains at different distances from interface: $50 \mu \mathrm{m}$ (a), $400 \mu \mathrm{m}$ (b), $500 \mu \mathrm{m}$ (c).

samples. An enhancement of the CL intensity is observed in the substrate as a bright layer of about $500 \mu \mathrm{m}$ thickness [Fig. 1(b)] except in some subsurface regions with dark contrast due to a high density of interface defects. CL images recorded inside this layer at higher magnification [Fig. 2(a)] reveal the presence of sub-boundaries and other structure defects as dark features on a bright background. A typical CL image of the substrate far from the modified layer is shown in Fig. 2(b). In this region, an inverse defect CL contrast relative to the modified layer is observed with bright subboundaries and precipitate like defects on a dark background. It should be noted that the substrates prior to the growth show no contrast reversal or enhancement in any areas but an appearance similar to the CL map of Fig. 2(b). The corresponding spectrum is shown in Fig. 3(a). As previously re- 
ported for Ge-doped CdTe, ${ }^{8}$ two bands centered at about 810 and $900 \mathrm{meV}$ are observed while the near band-edge emission is not detected. The spectra acquired by scanning the beam on a sub-boundary and on the background are qualitatively similar to some increase of the CL intensity on the sub-boundary. The $\alpha-\mathrm{HgI}_{2}$ epitaxial growth causes marked spectral changes in the CdTe substrate as shown by the CL spectrum recorded inside the modified layer [Fig. 3(b)]. This spectrum reveals an intense near band-edge emission at 1535 $\mathrm{meV}$ as well as bands at about 1050 and $780 \mathrm{meV}$.

In order to investigate in more detail the effect of the growth on the nature and spatial distribution of deep levels in the substrate, CL spectra were recorded at different distances from the interface (Fig. 4). It has been previously observed that by defocusing the electron beam the intensity of the main deep-level bands is enhanced relative to the near bandedge emission. For this reason a defocused electron beam was used when recording the spectra presented in Fig. 4. The series of Fig. 4 shows that the relative intensity of the three bands - 1070, 900 and $810 \mathrm{meV}$ - depends on the distance to the interface.

As stated above, the 810 and $900 \mathrm{meV}$ transitions are associated with defects involving Ge. ${ }^{8,15,16}$ The bright $\mathrm{CL}$ contrast of sub-boundaries and other defects in the unmodified part of the substrate indicates that extended structural defects are effective gettering sites for the Ge-related point defect complexes. Near the interface the relative contribution of the Ge-related defects to the luminescence decreases. Subboundaries and other features appear dark on a bright background. Spectra of the modified region indicate that the luminescence increase near the interface is due to increase of intensity of bands at about 1050 and $1540 \mathrm{meV}$. The former has been attributed to the presence of Te vacancies ${ }^{17,18}$ and the latter is not observed in Ge-doped crystals. ${ }^{8}$ Its appearance would be a consequence of the decrease of the active Ge-center concentration in the modified layer. A quenching of luminescence by the interface defects prevented the recording of CL spectra in an interface region of about $50 \mu \mathrm{m}$. However, the results obtained suggest a gettering of Ge defects at the $\alpha-\mathrm{HgI}_{2} / \mathrm{CdTe}$ interface or in the $\alpha-\mathrm{HgI}_{2}$ layer during the $\alpha-\mathrm{HgI}_{2}$ epitaxial growth. It should be noted that the diffusion of Te vacancies created at the interface alone could not induce the observed effect, in particular the near band-edge emission (the $1540 \mathrm{meV}$ band) enhancement (Fig. 3 ). The small shifts of the deep-level band peaks as a function of the distance to the interface and the excitation conditions (Fig. 3 and Fig. 4) indicate that the defects contributing to these complex bands have a concentration gradient relative to the interface or appear in different charge states. Defects involved in the $1050 \mathrm{meV}$ band peak shift are $V_{\mathrm{Te}}$ and/or residual impurities. It should be noted that the known thermal diffusion coefficients of iodine and mercury in $\mathrm{CdTe}^{19,20}$ at the time-temperature conditions of $\alpha-\mathrm{HgI}_{2}$ growth cannot explain the observed point defect effects in an extended - up to $500 \mu \mathrm{m}$-layer of the substrate.

In conclusion, it has been found that the overall CL emission of the CdTe:Ge substrate increases in a layer of about $500 \mu \mathrm{m}$ thickness as a result of epitaxial growth of $\alpha-\mathrm{HgI}_{2}$ at $250{ }^{\circ} \mathrm{C}$ for $24 \mathrm{~h}$. The appearance of band-edge emission and relative changes in the concentration of point defects related to $\mathrm{Ge}$ and of tellurium vacancies has been observed. Generation of the Te vacancies at interface and gettering of the Ge defects responsible of emission bands at 810 and $900 \mathrm{meV}$ take place during epitaxial growth. The present experiments do not yet permit us to determine the underlying mechanism which leads to the long range point defect distribution induced by $\alpha-\mathrm{HgI}_{2}$ epitaxial growth. Further studies have to be carried out for a better understanding of this phenomenon. Nevertheless, such substrate modifications extending a relatively long distance from the surface during epitaxial growth should be taken into account in the fabrication of $\alpha-\mathrm{HgI}_{2} / \mathrm{CdTe}$ detectors and other CdTe based optoelectronic devices.

G. P. and N. S. thank Spanish MEC for research grants. This work was supported by the DGICYT (Project No. PB 93-1256) and CICYT (Project No. ESP95-0148).

${ }^{1}$ M. Piechotka and E. Kaldis, Nucl. Instrum. Methods Phys. Res. A 283, 111 (1989).

${ }^{2}$ G. Entine, P. Waer, T. Tiernan, and M. R. Squillante, Nucl. Instrum. Methods Phys. Res. A 283, 282 (1989).

${ }^{3}$ V. I. Ivanov, V. A. Garbusin, P. G. Dorogov, A. E. Loutchanski, and V. V. Kondrashov, IEEE Trans. Nucl. Sci. 42, 258 (1995).

${ }^{4}$ Y. J. Wang, J. S. Iwanczyk, and B. E. Patt, IEEE Trans. Nucl. Sci. 41, 910 (1994).

${ }^{5}$ W. Dusi, E. Caroli, G. Di Cocco, A. Donati, G. Landini, G. Ramunno, M. Amann, J. M. Koebel, P. Siffert, D. Grassi, and E. Perillo, Nucl. Instrum. Methods Phys. Res. A 348, 531 (1994).

${ }^{6}$ M. G. Astles, Properties of Narrow Gap Cadmium-Based Compounds, edited by P. Capper (Institute of Electrical Engineers, London, 1994) pp. 494-500 and references therein.

${ }^{7}$ N. V. Sochinskii, M. D. Serrano, E. Dieguez, F. Agullo-Rueda, U. Pal, J. Piqueras, and P. Fernandez, J. Appl. Phys. 77, 2806 (1995).

${ }^{8}$ U. Pal, P. Fernandez, J. Piqueras, N. V. Sochinskii, and E. Dieguez, J. Appl. Phys. 78, 1992 (1995).

${ }^{9}$ U. Pal, J. Piqueras, M. D. Serrano, N. V. Sochinskii, and E. Dieguez, Appl. Phys. A 61, 645 (1995).

${ }^{10}$ N. V. Sochinskii, M. D. Serano, V. N. Babentsov, N. I. Tarbaev, J. Garrido, and E. Dieguez, Semicond. Sci. Technol. 9, 1713 (1994).

${ }^{11}$ N. V. Sochinskii, C. Marin, J. C. Rojo, and E. Dieguez (unpublished).

${ }^{12}$ F. Dominguez-Adame, J. Piqueras, and P. Fernandez, Appl. Phys. Lett. 58, 257 (1991).

${ }^{13}$ H. C. Casey and J. S. Jayson, J. Appl. Phys. 42, 2774 (1971).

${ }^{14}$ U. Pal, P. Fernandez, J. Piqueras, M. D. Serrano, and E. Dieguez, Inst. Phys. Conf. Ser. 135, 177 (1994)

${ }^{15}$ C. Scharager, P. Siffert, P. Hoschl, P. Moravec, and M. Vanecek, Phys. Status Solidi A 66, 87 (1981).

${ }^{16}$ W. Jantsch and G. Hendorfer, J. Cryst. Growth 101, 404 (1990).

${ }^{17}$ D. M. Hofmann, W. Stadler, K. Oettinger, B. K. Meyer, P. Omling, M. Salk, K. W. Benz, E. Wiegel, and G. Muller-Vogt, Mater. Sci. Eng. B 16, 128 (1993).

${ }^{18}$ F. J. Bryant and E. Webster, Phys. Status Solidi B 49, 499 (1972).

${ }^{19}$ J. C. Clark and E. D. Jones, Ref. 6, pp. 482-486.

${ }^{20}$ E. D. Jones and J. C. Clark, Ref. 6, pp. 472-481. 